\title{
Comprehensive immune profiling and immune-monitoring using body fluid of patients with metastatic gastric cancer
}

Hyung Soon Park ${ }^{1,2 \dagger}$, Woo Sun Kwon ${ }^{2,3 \dagger}$, Sejung Park ${ }^{2,3}$, Eunji Jo ${ }^{2,3}$, So Jung Lim² ${ }^{2}$ Choong-kun Lee ${ }^{4}$, Jii Bum Lee ${ }^{4}$, Minkyu Jung ${ }^{2,4}$, Hyo Song Kim ${ }^{2,4}$, Seung-Hoon Beom ${ }^{2,4}$, Jun Yong Park ${ }^{5}$, Tae Soo Kim², Hyun Cheol Chung 2,3,4 and Sun Young Rha $a^{2,3,4^{*}}$

\begin{abstract}
Background: The aim of this study is to profile the cytokines and immune cells of body fluid from metastatic gastric cancer (mGC), and evaluate the potential role as a prognostic factor and the feasibility as a predictive biomarker or monitoring source for immune checkpoint inhibitor.

Methods: Body fluid including ascites and pleural fluid were obtained from $55 \mathrm{mGC}$ patients and 24 matched blood. VEGF-A, LL-10, and TGF- $\beta 1$ were measured and immune cells were profiled by fluorescence assisted cell sorting (FACS).

Results: VEGF-A and IL-10 were significantly higher in body fluid than in plasma of mGC. Proportion of T lymphocytes with CD69 or PD-1, memory T cell marked with CD45RO, and number of Foxp3+ T regulatory cells (Tregs) were significantly higher in body fluid than those in blood of mGC. Proportion of CD8 T lymphocyte with memory marker (CD45RO) and activation marker (HLA-DR), CD3 T lymphocyte with PD-1, and number of FoxP3+ Tregs were identified as independent prognostic factors. When patients were classified by molecular subgroups of primary tumor, VEGF-A was significantly higher in genomically stable (GS)-like group than that in chromosomal instability (CIN)-like group while PD-L1 positive tumor cells (\%) showed opposite results. Monitoring immune dynamics using body fluid was also feasible. Early activated T cell marked with CD25 was significantly increased in chemotherapy treated group.
\end{abstract}

Conclusions: By analyzing cytokines and proportion of immune cells in body fluid, prognosis of patients with mGC can be predicted. Immune monitoring using body fluid may provide more effective treatment for patients with mGC.

Keywords: Cytokines, Immune profile, Body fluid, Metastatic gastric cancer

\section{Background}

Gastric cancer ranks the fourth among the most common cancers worldwide and the third in mortality rate [1]. It is the second most common cancer in Korea. About 30,000 new cases are diagnosed in 1 year [2]. About 30 35\% of gastric cancer patients show initial distant metastasis. Palliative chemotherapy is a standard treatment [3]. The survival of patients with metastatic gastric cancer (mGC) is less than 2 years. Anti-HER2 therapy with trastuzuamb

\footnotetext{
* Correspondence: rha7655@yuhs.ac

${ }^{+}$Hyung Soon Park and Woo Sun Kwon equally contributed as first author.

${ }^{2}$ Songdang Institute for Cancer Research, Yonsei University College of

Medicine, Seoul, South Korea

${ }^{3}$ Brain Korea 21 Project for Medical Sciences, Yonsei University College of

Medicine, Seoul, South Korea

Full list of author information is available at the end of the article
}

can prolong the survival of HER2 positive patients up to 13 months $[4,5]$. After the ToGA (Trastuzumab for Gastric Cancer) study, the first positive study in advanced gastric cancer using target agent, many studies have attempted to find targeted therapy according to molecular aberrations found in gastric cancer. Such studies are supported by genetic profiling of tumor in several groups including The Cancer Genome Atlas Research Network (TCGA) [6]. After failure of several kinds of targeted agents, immune checkpoint inhibitor (ICI) has emerged as a new treatment option for gastric cancer. Nivolumab and pembrolizumab have shown promising antitumor activity $[7,8]$. The biomarker of ICI is a major issue in cancer field including metastatic gastric cancer. It might help us better indicate which patients are most 
likely to have benefits [7,9]. Several biomarkers such as tumor mutation burden and programmed death-ligand 1 (PD-L1) status by immunohistochemistry (IHC) have been suggested. However, none of them is considered as a standard biomarker. The role of immune cells in immune checkpoint inhibitor response is well known, especially tumor infiltrating lymphocytes (TIL) in tumor microenvironment. Thus, tumor can be categorized as inflamed or non-inflamed. Inflamed tumor is characterized by the presence of TIL, high density of $\mathrm{CD} 8^{+} \mathrm{T}$ cell, and expression of PD-L1 in tumor or immune cells. Collective clinical evidence suggests that ICI is more effective for inflamed tumors [10].

However, not all patients with inflamed tumors respond to ICI. T cell receptor (TCR)-peptide-major histocompatibility complex (pMHC) binding is the central event in the activation of $\mathrm{T}$ cell. Activation antigens on $\mathrm{T}$ cell include CD25, CD26, CD38, CD54, CD69, and HLA-DR [11, 12]. Activated $\mathrm{T}$ cells can trigger TME evolution including upregulation of inflammatory/suppressive cytokines, immuneinhibitory cellular recruitment, and aberrant tumor vasculature related to innate/acquired resistance. The complex crosstalk among cancer cells, immune cells, and tumor microenvironment is closely connected to each other. Activation antigens on $\mathrm{T}$ cells could be detected according to time. CD69 and CD25 are early antigens whereas HLADR is a late marker [11]. The kinetics of expression of early activation markers (CD69 and CD25) was similar to that of PD-1 expression [13]. In tumor tissue, chronic exposure to antigen and the development of dysfunctional or exhausted effector $\mathrm{T}$ cell are accompanied by high expression of one or more inhibitory receptors including PD-1, lymphocyte activation gene 3 (LAG-3), and T-cell immunoglobulin and mucin-domain containing-3 (TIM-3) [14]. In addition, regulatory $\mathrm{T}$ cell and memory $\mathrm{T}$ cell play a role in the control of tumor growth and progression.

About $30-40 \%$ of mGC patients have malignant ascites associated with peritoneal carcinomatosis $[15,16]$. Ascites has a different tumor microenvironment from primary tumor, and it has high levels of immune suppressive cytokines and immune cells [17-19]. Especially, several cytokines such as VEGF-A, IL-10, and TGF- $\beta 1$ have immune suppressive function [20]. They are directly or indirectly related to angiogenesis which has close interaction with immune cells for immune surveillance [21, 22]. The role and profile of these cytokines have been mostly studied in ovarian cancer. Their expression level can affect patient prognosis and drug resistance [23]. However, little is known cytokines and immune signature in malignant ascites of gastric cancer. Only a small number of studies have dealt with this subject [20]. Ascites reflects tumor microenvironment. It has advantage such as easier acquisition than tumor biopsy. Malignant ascites contains several kinds of cytokines and plenty of immune cells having directly contact with tumor cells, suggesting that malignant ascites might serve as a good resource for immune monitoring of mGC patients. Therefore, the aim of this study was to obtain profiles of cytokines and immune cells of body fluid including ascites and pleural fluid in $\mathrm{mGC}$ and evaluate their potential roles as prognostic factors. The feasibility of using ascites as a predictive biomarker and a monitoring source for immune checkpoint inhibitor was also examined.

\section{Methods \\ Study population}

Between December 2014 and April 2016 at Yonsei Cancer Center, Seoul, Korea, body fluid including ascites and pleural fluid were obtained in a prospective, nonselective fashion from $55 \mathrm{mGC}$ patients via paracentesis or catheter drainage. Twenty-four matched blood samples were also taken before or at the time of body fluid acquisition. Cancer cells from body fluid were confirmed by pathologist using cell block. Eleven non-cancerous ascites and 4 matched blood samples were obtained from patients with Child-Pugh B/C liver cirrhosis. Blood samples from 15 healthy volunteers were used as controls. Clinical data including age and sex were collected for all study subjects. The following clinico-pathologic information was obtained by reviewing electronic medical records (EMR) of gastric cancer (GC) patients: disease presentation (recurrent or metastatic), type of surgery, differentiation, Lauren classification, HER2 status, type of body fluid, acquisition timing of body fluid, and survival time. This study was approved by the Institutional Review Board of Severance Hospital (No. 4-2014-0638).

\section{Measurement of immune suppressive cytokine}

VEGF-A, IL-10, and TGF- $\beta 1$ which are known immune suppressive cytokines were selected for measurement to evaluate their clinical roles and associations with immune cells. Plasma was isolated from collected $10 \mathrm{ml}$ peripheral blood in EDTA tube using Ficoll-Paque ${ }^{\mathrm{Tm}}$ PLUS (GE Healthcare, Sweden) gradient centrifugation and stored at $-80^{\circ} \mathrm{C}$ until assay. Body fluid was incubated in $10 \mathrm{X} \mathrm{RBC}$ lysis buffer (Biolegend, CA, USA) to remove RBC and centrifuged ( $5 \mathrm{~min}, 1500 \mathrm{rpm}$ ). The supernatant was separated, aliquoted, and stored at $-80^{\circ} \mathrm{C}$ until analysis. Plasma and body fluid supernatant were used to measure levels of circulating VEGF-A, IL-10, and TGF- $\beta 1$ using a commercially available enzyme-linked immunosorbent assay (ELISA) kit (Quantikine; R\&D Systems Abingdon, UK) following the manufacturer's protocols. Samples were measured in duplicates and mean value was presented as final concentration. ELISA plates were read on a Sunrise absorbance microplate reader (TECAN, Switzerland). 
Isolation of PBMC and lymphocyte in malignant fluid Peripheral blood mononuclear cells (PBMCs) were isolated from $10 \mathrm{ml}$ peripheral blood collected into EDTA tube using Ficoll-Paque ${ }^{\mathrm{Tx}}$ PLUS (GE Healthcare, Sweden) gradient centrifugation. Tumor associated lymphocytes (TALs) from 100 to $500 \mathrm{~mL}$ malignant fluid were isolated using the following protocol. Briefly, the fluid was incubated with $10 \mathrm{X}$ RBC lysis buffer (Biolegend, CA, USA) to remove $\mathrm{RBC}$ and centrifuged in $50 \mathrm{ml}$ tubes at $1500 \mathrm{rpm}$ for $5 \mathrm{~min}$. After two washes with PBS (phosphate buffered saline), isolated cells were suspended in $1 \mathrm{ml}$ of Cellbanker-2 (ZENOAQ, Japan) and stored at $-80^{\circ} \mathrm{C}$ until analysis.

\section{Flow cytometry and antibodies}

Flow cytometry was performed using FACS LSR2 (BD Biosciences, CA, USA). Data were analyzed using FlowJo software (FlowJo, LLC, OR, USA). Fluorescence-conjugated monoclonal antibodies were purchased from the following sources: Human LAG-3 Alexa Fluor ${ }^{\circ}$ 488-conjugated Antibody (R\&D system, MN, USA); PE/Cy7 anti-CD3, FITC anti-CD4, PE anti-CD8, FITC anti-CD45RO, FITC anti-HLA-DR, APC anti-CD25, APC/Cy7 anti-CD69, APC anti-human CD279 (PD-1), APC/Cy7 anti-human CD366 (Tim-3), and PE anti-FoxP3 (BioLegend, CA, USA). Furthermore, cells were stained with $\mathrm{PE}$ anti-human CD274 (B7-H1, PD-L1, BioLegend, CA, USA) to identify proportion of PD-L1 positive tumor cells in body fluid.

For Treg cell staining, cells were stained with various antibodies except FoxP3 antibody for which cells were fixed and permeabilized with eBioscience ${ }^{\text {nx }}$ FoxP3 Fixation/Permeabilization solution (Thermo Fisher Scientific, MA, USA). FoxP3 antibodies were administered after permeabilization for intracellular staining of Tregs. FACS analyses were performed for cells isolated from malignant fluid and peripheral blood. First, levels of CD4+ and $\mathrm{CD} 25+\mathrm{T}$ cells in cells isolated from these two sources (malignant fluid and peripheral blood) were measured. Next, we quantified the percentage of cells that were positive for FoxP3 in the CD4 + CD25+ T cell population.

\section{Molecular subtype of primary tumor by histochemistry}

Gastric cancer panel practically used in our institution has 10 markers, including Epstein-Barr virus encoded small RNAs (EBER) in-situ hybridization, mismatch repair proteins (MLH1, PMS2, MSH2, and MSH6), receptor tyrosine kinases (RTKs; HER2, EGFR, and MET), PTEN, and p53 protein expression by IHC using formalin-fixed, paraffinembedded (FFPE) tissue blocks from paired primary stomach cancer. Detailed methods of in situ hybridization (ISH) and IHC staining have been described in our previous study [24]. Patients were categorized by The Cancer Genome Atlas (TCGA) molecular subtypes, including Epstein Barr Virus (EBV) positive, microsatellite instability (MSI), genomically stable (GS)-like, and chromosomal instability (CIN)-like. CIN-like group patients had overexpression of HER2 (ISH 3+ or ISH2 + with amplification by ISH), EGFR ( $2+$ or $3+)$, MET ( $2+$ or $3+)$, and PTEN loss. Others with all markers negative were included in the GSlike group. In landscape analysis, continuous variables were expressed by categorized value (low vs. high group) which was determined by the best cut off point.

\section{Statistical analysis}

Differences in cytokine and immune profiles among blood and body fluid samples were compared using the MannWhitney U test. Pearson's correlation coefficient was calculated to determine relationships among variables. A value of higher than 0.7 means highly positive correlation [25]. Continuous variable were transformed into categorical variables with high or low to calculate the maximizing hazard ratio (HR) based on log-rank statistics presented by Contal and O'Quigley [26]. The overall survival (OS) was defined as the time from body fluid acquisition to death from any cause. Time to event endpoint was analyzed by KaplanMeier survival curves using the log-rank test. Scoring system using ascites cytokines according to best-cut off point was made to establish a prognostic model. A number of cytokines with high expression levels including VEGF-A, IL- 10 , and TGF- $\beta 1$ were categorized as $0-1$ and $2-3$. To determine independent prognostic factor, significant prognostic factors identified in univariate analysis were analyzed with multivariate Cox proportional hazard model using forward stepwise analysis. A p-value of less than 0.05 was considered statistically significant. PASW Statistics 18.0 (SPSS Inc. Chicago, IL, USA) was used for all statistical analyses.

\section{Results}

\section{Patient characteristics}

Baseline characteristics of enrolled patients are shown in Table 1 . The median age of 15 healthy volunteers was 63 years (range, 27-77 years) and eight (53\%) were females. Eleven Child-Pugh B/C liver cirrhosis patients with ascites were enrolled as controls. Their median age was 53 years (range, 35-79 years) and seven (63.6\%) were males. Fiftyfive mGC patients with ascites or pleural fluid were enrolled. Their median age was 58 years (range, 25-75). There were 66\% males. Fourteen (25\%) patients had recurrent gastric cancer. Thirty-nine (71\%) patients had poorly differentiated or signet ring cell features. Eight (14.5\%) patients were HER2 positive. Forty-four (80\%) patients had palliative chemotherapy history at the time of body fluid acquisition.

\section{Comparison of immune suppressive cytokines in healthy volunteers, liver cirrhosis patients, and gastric cancer patients}

Median values of plasma and body fluid cytokines are shown in Additional file 1: Table S1. Plasma VEGF-A 
Table 1 Baseline characteristics

\begin{tabular}{|c|c|c|c|}
\hline & & No. & $\%$ \\
\hline Healthy volunteers & $(n=15)$ & & \\
\hline Age & median, range & 63 & $27-77$ \\
\hline \multirow[t]{2}{*}{ Sex } & Male & 7 & 46.7 \\
\hline & Female & 8 & 53.3 \\
\hline Non-cancerous patients & $(n=11)$ & & \\
\hline Age & median, range & 53 & $35-79$ \\
\hline \multirow[t]{2}{*}{ Sex } & Male & 7 & 63.6 \\
\hline & Female & 4 & 36.4 \\
\hline Gastric cancer patients & $(n=55)$ & & \\
\hline Age & median, range & 58 & $25-75$ \\
\hline \multirow[t]{2}{*}{ Sex } & Male & 36 & 65.5 \\
\hline & Female & 19 & 34.5 \\
\hline \multirow[t]{2}{*}{ Disease presentation } & Recurrent & 14 & 25.5 \\
\hline & Metastatic & 41 & 74.5 \\
\hline \multirow[t]{3}{*}{ Type of surgery } & Total gastrectomy & 9 & 16.4 \\
\hline & Subtotal gastrectomy & 10 & 18.2 \\
\hline & No & 36 & 65.5 \\
\hline \multirow[t]{5}{*}{ Differentiation } & WD & 3 & 5.5 \\
\hline & MD & 9 & 16.4 \\
\hline & PD & 27 & 49.1 \\
\hline & SRC & 12 & 21.8 \\
\hline & Others $^{\mathrm{a}}$ & 4 & 7.3 \\
\hline \multirow[t]{4}{*}{ Lauren } & Intestinal & 7 & 12.7 \\
\hline & Diffuse & 10 & 18.2 \\
\hline & Mixed & 1 & 1.8 \\
\hline & Unknown & 37 & 67.3 \\
\hline \multirow[t]{3}{*}{ HER2 } & Negative & 46 & 83.6 \\
\hline & Positive & 8 & 14.5 \\
\hline & Unknown & 1 & 1.8 \\
\hline \multirow[t]{2}{*}{ Type of body fluid } & Ascites & 46 & 83.6 \\
\hline & Pleural fluid & 9 & 16.4 \\
\hline \multirow[t]{4}{*}{ Previous palliative chemotherapy at acquisition of body fluid } & Chemotherapy-naïve & 11 & 20.0 \\
\hline & 1 & 14 & 25.5 \\
\hline & 2 & 15 & 27.3 \\
\hline & $\geq 3$ & 15 & 27.3 \\
\hline
\end{tabular}

WD Well differentiated, $M D$ Moderate differentiated, $P D$ Poorly differentiated, SRC Signet ring cell carcinoma, No Number Others $^{\mathrm{a}}$ : mucinous adenocarcinoma, undifferentiated carcinoma

and IL-10 levels in mGC were significantly higher than those in healthy volunteers $(P=0.013$ and $P=0.001$, respectively). VEGF-A and IL-10 levels in mGC body fluid were significantly higher than those in mGC plasma and non-cancerous body fluid (Fig. 1a-b). In contrast, ascites TGF- $\beta 1$ levels in liver cirrhosis samples were lower than those in other samples, and remaining samples showed no significant difference in TGF- $\beta 1$ level among each other (Fig. 1c). When correlations of cytokines between plasma and body fluid in mGC were analyzed, levels of VEGF-A, but not those of IL-10 or TGF- $\beta 1$, showed significant correlations $(P=0.004$, correlation coefficient, $r=0.5647$ ) (Additional file 4: Figure S1). In addition, there were no significant correlations among cytokines in body fluid (Additional file 5: Figure S2). 

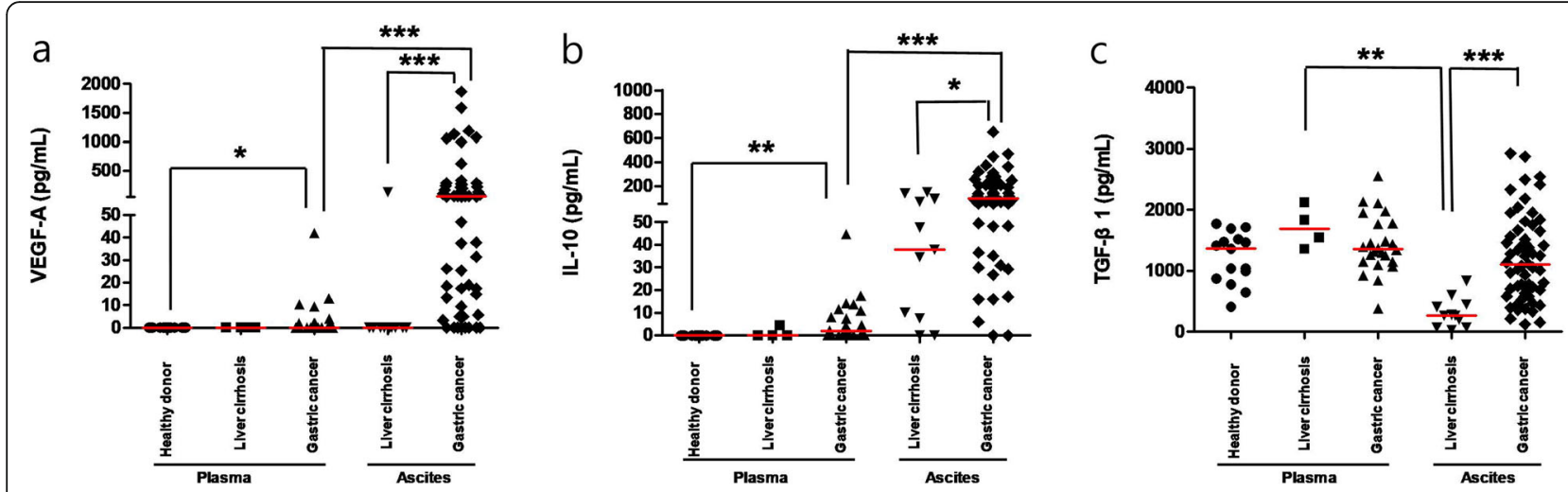

Fig. 1 Pro-angiogenic, immune modulatory cytokine, and immunosuppressive cytokines have different patterns in plasma and body fluid among healthy volunteer, non-cancerous patients, and gastric cancer patients. a Plasma VEGF-A in mGC were significantly higher than those in healthy volunteers $(P=0.013)$. VEGF-A levels in $\mathrm{mGC}$ body fluid were significantly higher than those in mGC plasma and non-cancerous body fluid. $\mathbf{b}$ Plasma IL-10 levels in mGC were significantly higher than those in healthy volunteers $(P=0.001)$. IL-10 levels in mGC body fluid were significantly higher than those in mGC plasma and non-cancerous body fluid ( $P=0.014)$. c TGF- $\beta 1$ levels in non-cancerous body fluid were significantly lower than those in non-cancerous plasma ( $P=0.005)$ and $\mathrm{mGC}$ body fluid. $\mathrm{mGC}$, metastatic gastric cancer. Red line indicates median value. MannWhitney $U$ test was used for statistical analysis. ${ }^{*} P<0.05,{ }^{* *} P<0.01{ }^{* * *} P<0.001$

\section{Immune cell profiling of paired PBMC and body fluid in} mGC

Results of immune cell profiling of peripheral blood and body fluid in $\mathrm{mGC}$ are summarized in Additional file 2: Table S2. Proportion of CD8/CD3 $\mathrm{T}$ cells showed a higher tendency while CD4/CD8 ratio showed a lower tendency in body fluid than those in peripheral blood ( $P=0.073$ and $P=0.075$, respectively) of mGC. Proportion of memory $\mathrm{T}$ cell marked with CD45RO (CD3CD45RO, CD4CD45RO and CD8CD45RO) and activated $\mathrm{T}$ lymphocytes (early activation marker CD3CD69, CD4CD69, CD8CD69; late activation marker CD4HLADR) were significantly higher in body fluid than those in peripheral blood of mGC (Fig. 2a, b). In addition, T lymphocytes with inhibitory marker including PD-1 (CD3PD1, CD4PD1 and CD8PD1) and number of FoxP3+ $\mathrm{T}$ regulatory cells (Tregs) were significantly higher in body fluid than those in peripheral blood (Fig. 2c-d). Higher number of FoxP3+ Tregs in body fluid was significantly associated with increased $\mathrm{T}$ cell with inhibitor marker such as LAG3 and TIM3 (Additional file 6: Figure S3).

We then compared levels of cytokines and immune cell profiling. VEGF-A and proportion of $\mathrm{T}$ cells with CD69 or CD25 showed significant positive correlations (CD3CD69, correlation coefficient, $r=0.377, P=0.021$; CD4CD69, $\mathrm{r}=0.374, P=0.023 ; \quad \mathrm{CD} 4 \mathrm{CD} 25, \mathrm{r}=0.357$, $P=0.03 ; \mathrm{CD} 8 \mathrm{CD} 25, \mathrm{r}=0.688, P<0.001)$. In contrast, VEGF-A and number of FoxP3+ Tregs showed significant negative correlation $(\mathrm{r}=-0.339, P=0.043)$ (Additional file 7: Figure S4), suggesting that increased VEGF-A level might be related to immune suppressive microenvironment.
Survival analysis for immune monitoring as a prognostic factor

In survival analysis based on cytokines, high level of each cytokine in body fluid (VEGF-A, IL-10, or TGF- $\beta 1$ ) showed poor survival outcome with borderline tendency (Data not shown). However, patients with high levels of at least two cytokines showed significantly shorter OS than patients with zero or one cytokine at high level (median OS, 1.6 vs. 2.2 months, $P=0.032$, Fig. 3a-b).

Survival analysis was also conducted using immune cell profiles. Results are shown in Fig. 3c-f. Higher proportions of CD4/CD8 ratio, memory $\mathrm{T}$ cells (CD3CD45RO, CD4CD45RO, CD8CD45RO), and T lymphocytes with activation marker (CD3HLA-DR, CD4HLA-DR, CD8HLADR, CD4CD25) or inhibitory marker (CD3PD1, CD8PD1) were significantly associated with poor prognosis in univariate analysis (Table 2 and Additional file 3: Table S3). In multivariate analysis, proportion of CD8 T lymphocyte with memory marker (CD8CD45RO) and late activation marker (CD8HLA-DR), CD3 T lymphocyte with PD-1 (CD3PD1), number of FoxP3+ Tregs, and previous palliative chemotherapy history remained as independent prognostic factors (Table 2).

\section{Landscape analysis according to immune profile of body fluid in each molecular subtype}

Patients were classified by molecular subgroups of primary tumor. Patterns of cytokine and immune cell profile from body fluid were compared with molecular subgroups (Fig. 4). Only 1 patient was included in EBV positive and MSI group, respectively. Thus, we focused on CIN-like group (defined as those with overexpression of RTKs) and GS-like group (defined as those who were 


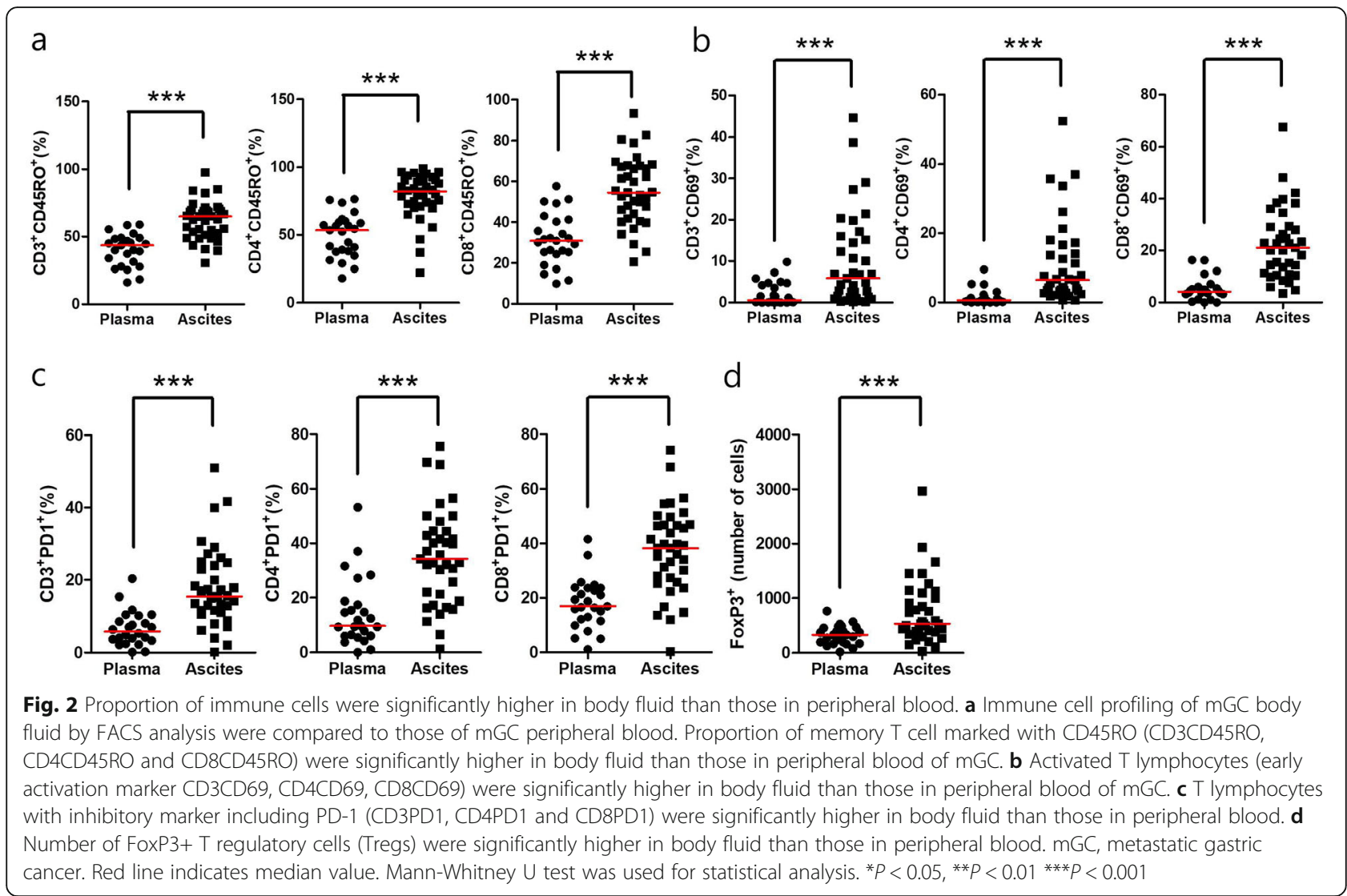

negative for all markers including EBV, microsatellite, and RTKs) to evaluate differences in cytokine and immune profiling. VEGF-A level was significantly higher in GS-like group than that in CIN-like group (median value: 163.9 vs. $17.4 \mathrm{pg} / \mathrm{mL}, P=0.003$ ). No significant difference was observed in immune cell profile, although percentage of PD-L1 positive tumor cells showed a higher tendency in CIN-like group than that in GS-like group (median proportion: 0.47 vs. $0.17 \%, P=0.08$ ) (Fig. $5 \mathrm{a}$-b).

\section{Dynamics of immune profile by chemotherapy}

Cytokine and immune cell profile from body fluid were compared between chemotherapy naïve and previously treated groups. Proportion of early activated $\mathrm{T}$ cells (CD3CD25 and CD4CD25) in previously chemotherapy treated patients was higher level than that in chemotherapy naive patients $(P=0.017$ and $P=0.035$, respectively, Fig. $5 \mathrm{c}-\mathrm{d}$ ). Fraction of memory $\mathrm{T}$ cell with CD45RO showed higher tendency in chemotherapy treated patients than that in chemotherapy naive patients (CD3CD45, $P=0.084$; CD8CD45, $P=0.157$ ). In addition, body fluid of patients treated with chemotherapy had higher VEGF-A levels than chemotherapy naïve patients (median value, 59.7 vs. $31.4 \mathrm{pg} / \mathrm{mL}, P=0.535$ ), suggesting that chemotherapy might induce immune suppressive environment.

\section{Discussion}

Malignant body fluid of mGC has diverse cytokines and immune cells which can represent tumor microenvironment. It is relatively easy to access. In this study, immune suppressive cytokines of malignant ascites were increased compared to those of non-cancerous ascites. These cytokines are significantly associated with diverse subsets of immune cells. Immune cells with CD8CD45RO, CD8HLA-DR, CD3PD1, and FoxP3+ Tregs had a prognostic role in $\mathrm{mGC}$. In addition, cytokine and immune cell profiles of body fluid were different according to molecular subtype of primary tumor and they can be changed by cytotoxic chemotherapy.

As an extension of this study, in real-world practice, angiogenesis inhibitor such as ramucirumab which had inhibitory mechanism of interaction between VEGFR2 and VEGFs [27] could be preferred for mGC with higher level of VEGF-A in malignant body fluid at any time point. In addition, ICI could be given to patients with lower level of VEGF-A (those of CIN-like group which had higher percentage of PD-L1 positive tumor cells), despite the lack of strong evidence. In the near future, biomarker studies can be performed for mGC patients who have developed malignant body fluid to evaluate the role of VEGF-A as a predictive marker for angiogenesis inhibitor or immune checkpoint inhibitor in randomized clinical trials. 


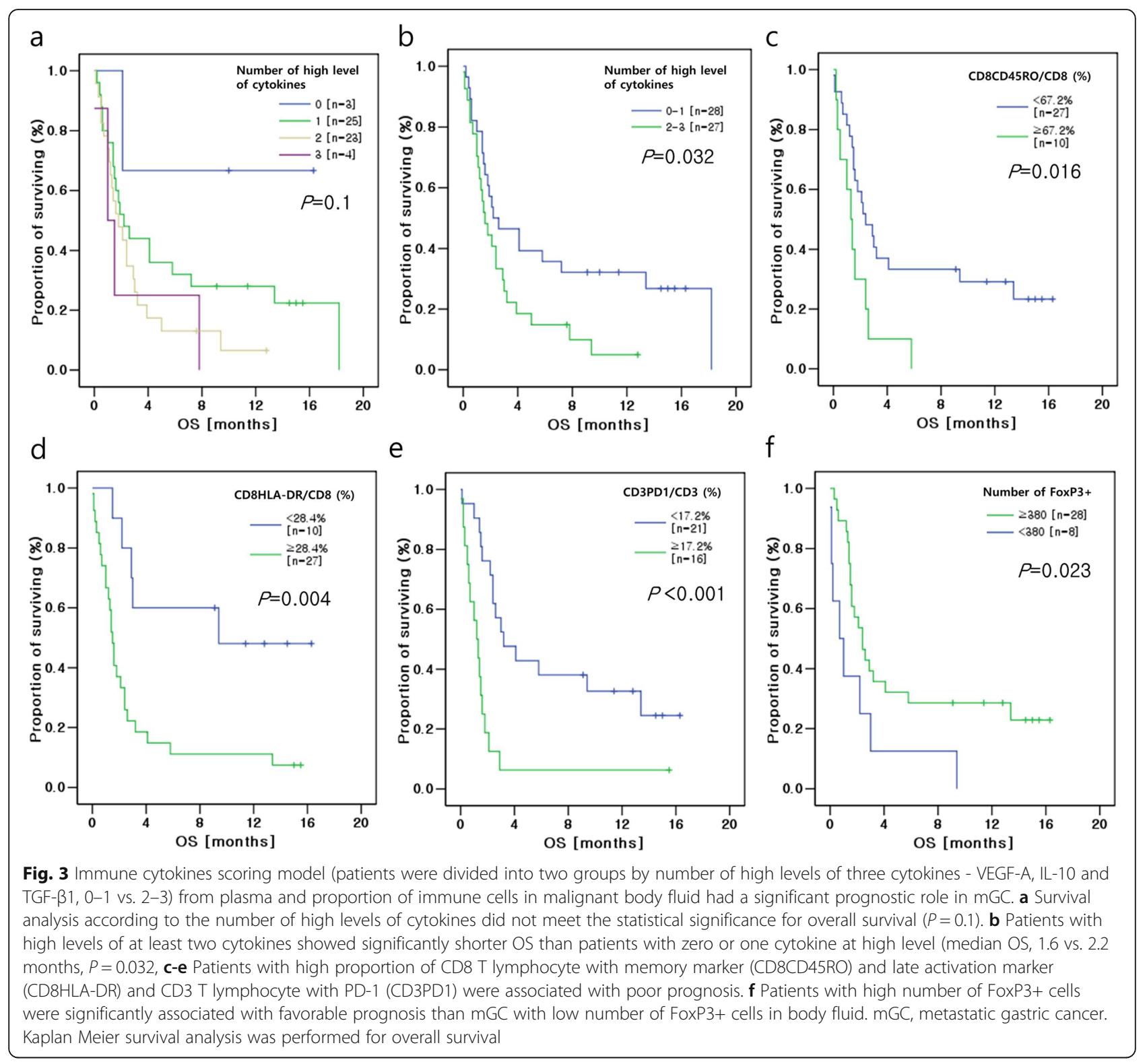

Tumor secrets various immune suppressive cytokines such as VEGF, IL-6, and IL-10 to promote the accumulation of heterogeneous populations of tumor associated macrophages (TAMs), myeloid-derived suppressor cells (MDSCs), and regulatory T cells [28]. VEGF-A, IL-10, and TGF- $\beta 1$ have been previously studied in metastatic ovarian cancer patients with ascites. Most of these studies showed that patients with higher levels of VEGF-A and IL10 had adverse prognosis [19, 29]. Likewise, our study showed that patients with higher levels of VEGF-A and IL-10 had poor prognostic tendency. TGF- $\beta 1$ of body fluid was revealed as a poor prognostic factor in this study for the first time. In addition, the lowest level of TGF- $\beta 1$ was observed in non-cancerous body fluid. This suggests that TGF- $\beta 1$ also has biological role in non-cancerous body fluid. Using body fluid cytokines, we made a scoring model to predict prognosis of patients with $\mathrm{mGC}$, but it should be validated by further studies.

Immune cell profiling of body fluid in mGC was also conducted and compared to that of plasma. Proportion of memory $\mathrm{T}$ cell with CD45RO and early activated $\mathrm{T}$ cell with CD69 was higher in malignant body fluid than that in plasma. This phenomenon was observed in other studies on ovarian cancer [30]. It might be related to malignant cells of body fluid that can induce immunogenicity. Proportions of suppressive $\mathrm{T}$ cell with PD-1 (CD3PD1, CD4PD1 and CD8PD1) and FoxP3+ Tregs were also increased in malignant body fluid. The mechanism of these phenomena could not be explained exactly. We can hypothesize that tumor cells in body 
Table 2 Univariate analysis for overall survival

\begin{tabular}{|c|c|c|c|c|c|c|c|c|c|}
\hline \multirow{3}{*}{ Age (years) } & & \multicolumn{4}{|c|}{ Univariate analysis } & \multicolumn{4}{|c|}{ Multivariate analysis } \\
\hline & & \multirow{2}{*}{$\begin{array}{l}\mathrm{HR} \\
1\end{array}$} & \multicolumn{2}{|c|}{$95 \% \mathrm{Cl}$} & \multirow{2}{*}{$\begin{array}{l}P \\
0.913\end{array}$} & \multirow[t]{2}{*}{$\mathrm{HR}$} & \multicolumn{2}{|c|}{$95 \% \mathrm{Cl}$} & \multirow[t]{3}{*}{ P } \\
\hline & $<58$ years & & & & & & & & \\
\hline & $\geq 58$ years & 1.03 & 0.57 & 1.86 & & & & & \\
\hline \multirow[t]{2}{*}{ Sex } & Male & 1 & & & 0.831 & & & & \\
\hline & Female & 0.94 & 0.51 & 1.73 & & & & & \\
\hline \multirow[t]{2}{*}{ Disease presentation } & Recurrent & 1 & & & 0.327 & & & & \\
\hline & Metastatic & 1.42 & 0.70 & 2.89 & & & & & \\
\hline \multirow[t]{5}{*}{ Differentiation } & WD & 1 & & & 0.602 & & & & \\
\hline & $\mathrm{MD}$ & 4.94 & 0.61 & 39.76 & & & & & \\
\hline & PD & 4.41 & 0.59 & 32.82 & & & & & \\
\hline & SRC & 3.41 & 0.44 & 26.72 & & & & & \\
\hline & Others* & 3.64 & 0.38 & 35.20 & & & & & \\
\hline \multirow[t]{2}{*}{ HER2 } & Negative & 1 & & & 0.892 & & & & \\
\hline & Positive & 0.81 & 0.34 & 1.93 & & & & & \\
\hline \multirow[t]{2}{*}{ Palliative chemotherapy history } & No & 1 & & & 0.003 & 1 & & & 0.005 \\
\hline & Yes & 4.19 & 1.63 & 10.78 & & 10.78 & 2.05 & 56.81 & \\
\hline \multirow[t]{5}{*}{ Molecular subtype of primary tumor } & MSI & 0.87 & 0.11 & 6.64 & 0.143 & & & & \\
\hline & EBV & 19.11 & 1.90 & 192.25 & & & & & \\
\hline & CIN-like & 1.01 & 0.49 & 2.07 & & & & & \\
\hline & GS-like & 1 & & & & & & & \\
\hline & Unknown & 1.33 & 0.63 & 2.84 & & & & & \\
\hline \multirow[t]{2}{*}{ VEGF-A (pg/mL) } & $<100.9 \mathrm{pg} / \mathrm{mL}$ & 1 & & & 0.127 & & & & \\
\hline & $\geq 100.9 \mathrm{pg} / \mathrm{mL}$ & 1.58 & 0.88 & 2.86 & & & & & \\
\hline \multirow[t]{2}{*}{$\mathrm{IL}-10(\mathrm{pg} / \mathrm{mL})$} & $>243.8 \mathrm{pg} / \mathrm{mL}$ & 1 & & & 0.16 & & & & \\
\hline & $\geq 243.8 \mathrm{pg} / \mathrm{mL}$ & 1.64 & 0.82 & 3.25 & & & & & \\
\hline \multirow[t]{2}{*}{ TGF- $\beta 1(p g / m L)$} & $<393.6 \mathrm{pg} / \mathrm{mL}$ & 1 & & & 0.197 & & & & \\
\hline & $\geq 393.6 \mathrm{pg} / \mathrm{mL}$ & 1.97 & 0.70 & 5.55 & & & & & \\
\hline Cytokine-scoring model & $0-1$ & 1 & & & 0.037 & & & & \\
\hline (number of high level of cytokines) & $2-3$ & 1.91 & 1.04 & 3.49 & & & & & \\
\hline \multirow[t]{2}{*}{ CD4/CD3 (\%) } & $<41.7 \%$ & 1 & & & 0.232 & & & & \\
\hline & $\geq 41.7 \%$ & 0.65 & 0.32 & 1.32 & & & & & \\
\hline \multirow[t]{2}{*}{ CD8/CD3 (\%) } & $<33.3 \%$ & 1 & & & 0.272 & & & & \\
\hline & $\geq 33.3 \%$ & 1.58 & 0.70 & 3.57 & & & & & \\
\hline \multirow[t]{2}{*}{ CD4/CD8 ratio } & $<0.6$ & 1 & & & 0.034 & & & & \\
\hline & $\geq 0.6$ & 0.35 & 0.13 & 0.92 & & & & & \\
\hline \multirow[t]{2}{*}{ CD8CD45RO+/CD8 (\%) } & $<67.2 \%$ & 1 & & & 0.021 & 1 & & & 0.019 \\
\hline & $\geq 67.2 \%$ & 2.54 & 1.15 & 5.59 & & 3.25 & 1.22 & 8.68 & \\
\hline CD8CD25+/CD8 (\%) & $<1.6 \%$ & 1 & & & 0.081 & & & & \\
\hline & $\geq 1.6 \%$ & 1.92 & 0.92 & 3.99 & & & & & \\
\hline CD8CD69+/CD8 (\%) & $<28.0 \%$ & 1 & & & 0.054 & & & & \\
\hline & $\geq 28.0 \%$ & 2.09 & 0.99 & 4.41 & & & & & \\
\hline CD8HLA-DR+/CD8 (\%) & $<28.4 \%$ & 1 & & & 0.008 & 1 & & & 0.012 \\
\hline & $\geq 28.4 \%$ & 3.77 & 1.43 & 9.96 & & 5.36 & 1.45 & 19.88 & \\
\hline
\end{tabular}


Table 2 Univariate analysis for overall survival (Continued)

\begin{tabular}{|c|c|c|c|c|c|c|c|c|c|}
\hline & & \multicolumn{4}{|c|}{ Univariate analysis } & \multicolumn{4}{|c|}{ Multivariate analysis } \\
\hline & & $\mathrm{HR}$ & $95 \% \mathrm{C}$ & & $P$ & $\mathrm{HR}$ & $95 \%$ & & $P$ \\
\hline \multirow[t]{2}{*}{ CD8 TIM3+/CD8 (\%) } & $<0.2 \%$ & 1 & & & 0.082 & & & & \\
\hline & $\geq 0.2 \%$ & 0.50 & 0.23 & 1.09 & & & & & \\
\hline \multirow[t]{2}{*}{ CD8 LAG3+/CD8 (\%) } & $<3.7 \%$ & 1 & & & 0.098 & & & & \\
\hline & $\geq 3.7 \%$ & 1.92 & 0.89 & 4.17 & & & & & \\
\hline \multirow[t]{2}{*}{ CD8 PD1+/CD8 (\%) } & $<50.2 \%$ & 1 & & & 0.038 & & & & \\
\hline & $\geq 50.2 \%$ & 2.58 & 1.06 & 6.31 & & & & & \\
\hline \multirow[t]{2}{*}{ CD3 PD1+/CD3 (\%) } & $<17.2 \%$ & 1 & & & 0.001 & 1 & & & $<0.001$ \\
\hline & $\geq 17.2 \%$ & 3.53 & 1.66 & 7.52 & & 8.61 & 2.81 & 26.37 & \\
\hline \multirow[t]{2}{*}{ CD4 PD1+/CD4 (\%) } & $<32.1 \%$ & 1 & & & 0.052 & & & & \\
\hline & $\geq 32.1 \%$ & 2.11 & 0.99 & 4.50 & & & & & \\
\hline \multirow[t]{2}{*}{ FoxP3+ Tregs (No) } & $<380$ & 1 & & & 0.029 & 1 & & & 0.001 \\
\hline & $\geq 380$ & 0.40 & 0.18 & 0.91 & & 0.13 & 0.04 & 0.42 & \\
\hline \multirow[t]{2}{*}{ PD-L1 positive tumor cells (\%) } & $<1 \%$ & 1 & & & 0.87 & & & & \\
\hline & $\geq 1 \%$ & 1.07 & 0.49 & 2.34 & & & & & \\
\hline
\end{tabular}

fluid might induce body fluid to have an immune suppressive status while activation marker is increased by compensatory mechanism.

As a prognostic factor, higher proportions of CD8CD45RO, CD8HLA-DR, and CD3PD1 were independent poor prognostic factors. CD45RO and HLA-DR are generally regarded as activation markers while PD1 is a well-known suppressive marker [31, 32]. The number of FoxP3+ Tregs was an independent prognostic factor in our study, with higher number showing favorable prognostic factor. Many studies have reported the role of FoxP3+ Tregs [33]. However, prognostic values of these cells in cancer remain controversial. FoxP3+ Tregs are associated with short survival in the majority of solid tumors including melanomas, cervical, renal, and breast cancers. In contrast, FoxP3+ Tregs are associated with improved survival in colorectal and esophageal cancer [33]. It has been suggested that the role of FoxP3+ Tregs is influenced by tumor sites, molecular subtypes, and tumor stage, although related mechanisms are currently unknown.

Molecular subtype was classified into MSI, EBV, CINlike, and GS-like group based on immunohistochemistry results, not genomics. Differences in cytokine and immune profiling were clustered by each group. VEGF-A level showed significant difference between CIN-like and GS-like group. VEGF-A level was higher in the GS-like group. However, other factors did not show statistically significant differences between these two groups. CIN-like group showed increased tendency of proportion of PD-L1 positive cancer cells than that in GS-like group. According to these differences, anti-VEGF therapy might give GSlike group more benefit while PD1 or PD-L1 inhibitor can be applied to CIN-like group. However, further mechanisms and clinical studies are needed to establish precision medicine based on body fluid monitoring.

The benefit of immune monitoring has already been studied and several approaches including tissue biopsy and blood sampling are ongoing [10,34,35]. Body fluid acquisition is more accessible than tissue. Thus, it is more useful as a source of predictive marker. In addition, it can be evaluated in real-time. To observe dynamics of cytokines and immune profile, we evaluated differences in cytokines and immune profile between chemotherapy naive and treated patients. Early activated $\mathrm{T}$ cell marked with CD25 (CD3CD25, CD4CD25), memory T cell with CD45RO, and VEGF-A were higher in previously chemotherapy treated group. Immune suppressive status after chemotherapy can be assumed. By real-time monitoring of the immune environment of patients, more effective treatment strategy can be applied.

This study has some limitations. Firstly, a small number of patients were analyzed. Therefore, it is hard to have enough statistical power. Results of this study should be validated through more studies with larger sample size. Second, we only checked a limited number of cytokines and immune cell markers. This should be overcome by multiplex technology including cytometry by time of flight (CyTOF). In addition, we did not perform comparison between body fluid and tumor tissues due to invasiveness of tumor biopsy and poor performance of most patients. 


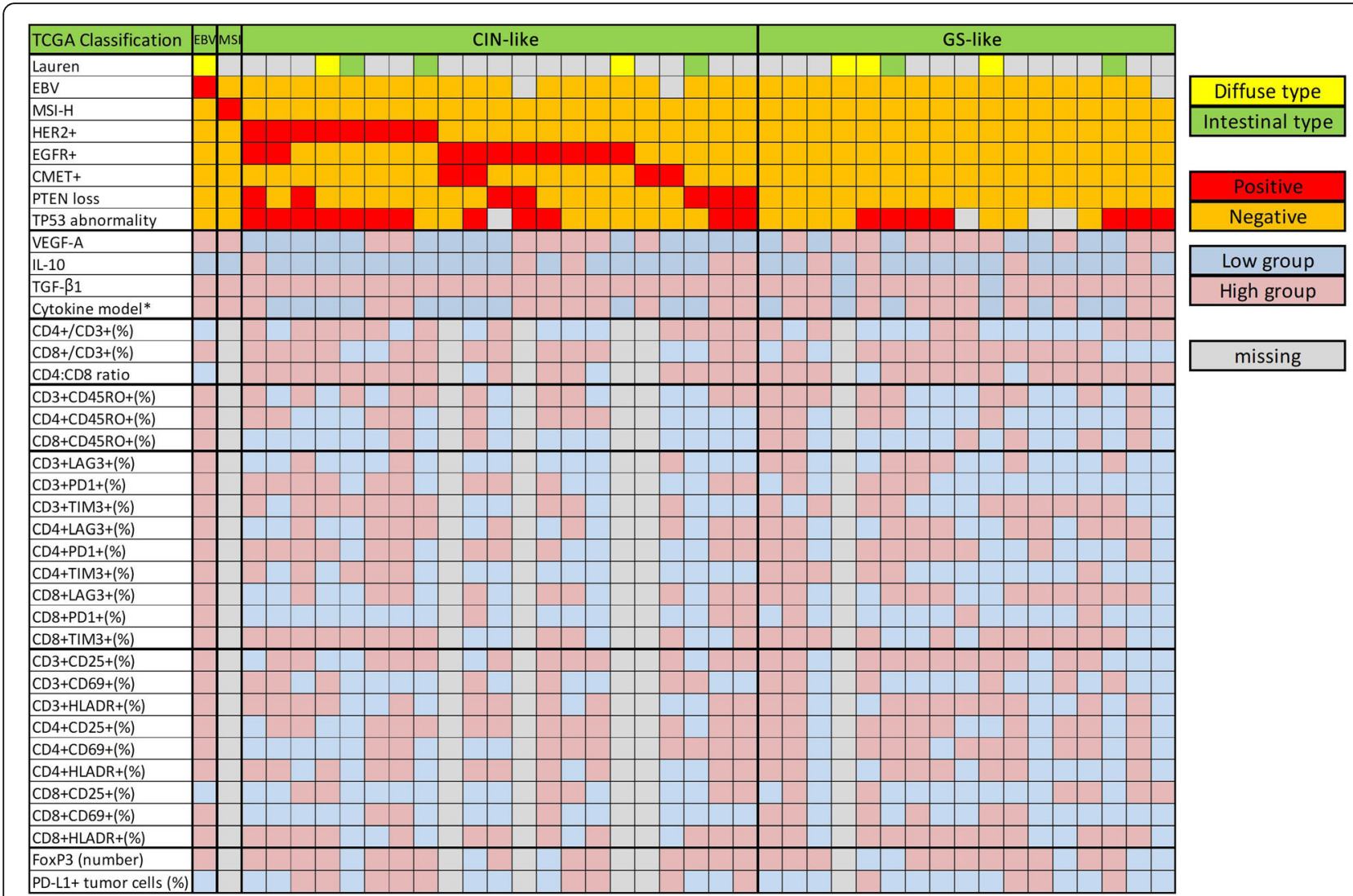

Fig. 4 Landscape shows the immune signature of body fluid in each molecular subtype of primary tissue. Patients were categorized by The Cancer Genome Atlas (TCGA) molecular subtype which was composed of Epstein Barr Virus (EBV) positive, microsatellite instability (MSI), genomically stable (GS)-like and chromosomal instability (CIN)-like. Diffuse type and intestinal type by Lauren classification were filled with yellow and green, respectively. Molecular markers by histochemistry were classified with positive (red) or negative (orange). Continuous value of cytokine and immune cell proportion were dichotomized by best-cut off which calculates the maximizing hazard ratio (HR) based on log-rank statistics (low vs. high). Low value was filled with blue while high value was filled with pink. * Cytokine model was scored by a number of high levels of VEGF-A, IL-10 and TGF- $\beta 1$, and it was categorized 0-1 (low group) and 2-3 (high group). TCGA, The Cancer Genome Atlas; EBV, Epstein-Barr virus; MSI-H, microsatellite instability-high; CIN, chromosomal instability; GS, genomically stable; VEGF, vascular endothelial growth factor; IL, interleukin; TGF- $\beta 1$, transforming growth factor- beta1; CD, cluster of differentiation

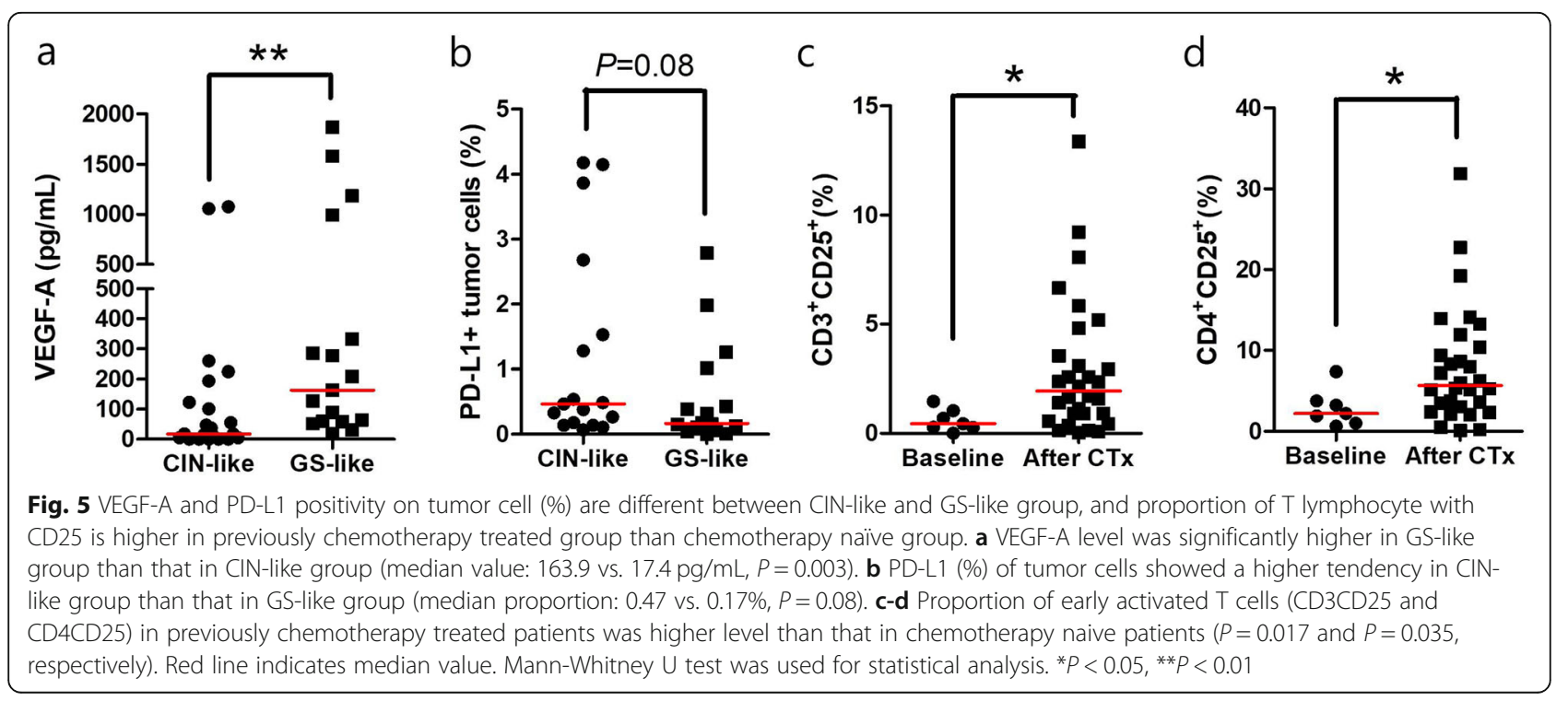


However, this study also has several advantages. Healthy volunteer and non-cancerous patients with ascites were included as controls to find the distinct meaning of immune profiling from malignant body fluid. In most studies, characterization and prognostic role of malignant body fluid were reported in ovarian cancer. Study of mGC body fluid was limited. We comprehensively characterized cytokines and immune profile of body fluid and evaluated the possibility of using body fluid as a monitoring source for predicting prognosis and marking therapeutic decision. In addition, we checked the proportion of PD-L1 positive tumor cells in body fluid. However, further studies with serial sampling of body fluid acquisition and immune profiling of paired primary tumor tissue are needed to verify our results.

\section{Additional files}

Additional file 1: Cytokine level of plasma and body fluid in healthy volunteers, non-cancerous and gastric cancer patients. (DOCX $16 \mathrm{~kb}$ )

Additional file 2: Immune cell profiling of peripheral blood and body fluid in metastatic gastric cancer patients. (DOCX $20 \mathrm{~kb}$ )

Additional file 3: Univariate analysis of body fluids immune cell subset for overall survival. (DOCX $20 \mathrm{~kb}$ )

Additional file 4: Correlation of cytokine levels between plasma and body fluid. (PPTX $80 \mathrm{~kb}$ )

Additional file 5: Correlation of different cytokines in body fluid. (PPTX $81 \mathrm{~kb})$

Additional file 6: Correlation between FoxP3+ T regulatory cells (Tregs) and immune cells with suppressive markers. (PPTX $147 \mathrm{~kb}$ )

Additional file 7: Correlation between VEGF-A and proportion of immune cells in the body fluid. (PPTX $190 \mathrm{~kb}$ )

\section{Abbreviations}

CD: Cluster of Differentiation; Cl: Confidence Interval; CIN: Chromosomal Instability; EBV: Epstein-Barr Virus; GS: Genomically Stable; HLA: Human Leukocyte Antigen; HR: Hazard Ratio; IL: Interleukin; MD: Moderate Differentiated; mGC: Metastatic Gastric Cancer; MSI: Microsatellite Instability; P: P-Value; PD-1: Programmed Death 1; PD-L1: Programmed Death Ligand 1; SRC: Signet Ring Cell; TCGA: The Cancer Genome Atlas; TGF-

B1: Transforming Growth Factor-Beta 1; VEGF: Vascular Endothelial Growth Factor; WD: Well Differentiated

\section{Acknowledgement}

The authors would like to thank you for our patients.

\section{Authors' contributions}

HP analyzed the data and wrote the manuscript. WK performed experiments and wrote the manuscript. SP analyzed the data. EJ and SL performed experiments. $C L$ reviewed the manuscript. JL, MJ, HK, SB, JP treated patients and we could acquire the body fluid from these patient. TK performed experiments $\mathrm{HC}$ made intellectual contributions to the method. SR suggested the project, made intellectual contributions to the analyses, and helped in writing the manuscript. All authors read and approved the final manuscript.

\section{Funding}

This research was supported by a grant of the Korea Health Technology R\&D Project through the Korea Health Industry Development Institute (KHIDI) funded by the Ministry of Health \& Welfare, Republic of Korea (H113C2096) and Basic Science Research Program through the National Research Foundation of Korea (NRF) funded by the Ministry of Science, ICT \& Future Planning (2017R1A2B2005772).

\section{Availability of data and materials}

The datasets used and/or analyzed during the current study are available from the corresponding author on reasonable request.

\section{Ethics approval and consent to participate}

This study was approved by the Institutional Review Board of Severance Hospital (No. 4-2014-0638).

\section{Consent for publication}

Not applicable.

\section{Competing interests}

The authors declare that they have no competing interests.

\section{Author details}

'Division of Medical Oncology, Department of Internal Medicine, St. Vincent's Hospital, The Catholic University of Korea, Suwon, South Korea. ${ }^{2}$ Songdang Institute for Cancer Research, Yonsei University College of Medicine, Seoul, South Korea. ${ }^{3}$ Brain Korea 21 Project for Medical Sciences, Yonsei University College of Medicine, Seoul, South Korea. ${ }^{4}$ Division of Medical Oncology, Department of Internal Medicine, Yonsei Cancer Center, Yonsei University College of Medicine, 50-1 Yonsei-Ro, Seodaemun-gu, Seoul 120-752, South Korea. ${ }^{5}$ Division of Gastroenterology, Department of Internal Medicine, Yonsei University College of Medicine, Seoul, South Korea.

Received: 5 February 2019 Accepted: 14 August 2019

Published online: 21 October 2019

\section{References}

1. Torre LA, Bray F, Siegel RL, Ferlay J, Lortet-Tieulent J, Jemal A. Global cancer statistics, 2012. CA Cancer J Clin. 2015;65(2):87-108.

2. Oh CM, Won YJ, Jung KW, Kong HJ, Cho H, Lee JK, et al. Cancer statistics in Korea: incidence, mortality, survival, and prevalence in 2013. Cancer Res Treat. 2016;48(2):436-50.

3. Ebinger SM, Warschkow R, Tarantino I, Schmied BM, Guller U, Schiesser M. Modest overall survival improvements from 1998 to 2009 in metastatic gastric cancer patients: a population-based SEER analysis. Gastric Cancer. 2016;19(3):723-34

4. Bang YJ, Van Cutsem E, Feyereislova A, Chung HC, Shen L, Sawaki A, et al. Trastuzumab in combination with chemotherapy versus chemotherapy alone for treatment of HER2-positive advanced gastric or gastrooesophageal junction cancer (ToGA): a phase 3, open-label, randomised controlled trial. Lancet. 2010;376(9742):687-97.

5. Lee JH, Kim JG, Jung HK, Kim JH, Jeong WK, Jeon TJ, et al. Clinical practice guidelines for gastric cancer in Korea: an evidence-based approach. J Gastric Cancer. 2014;14(2):87-104.

6. Network TCGAR. Comprehensive molecular characterization of gastric adenocarcinoma. Nature. 2014:513(7517):202-9.

7. Kang YK, Boku N, Satoh T, Ryu MH, Chao Y, Kato K, et al. Nivolumab in patients with advanced gastric or gastro-oesophageal junction cancer refractory to, or intolerant of, at least two previous chemotherapy regimens (ONO-4538-12, ATTRACTION-2): a randomised, double-blind, placebocontrolled, phase 3 trial. Lancet. 2017;390(10111):2461-71.

8. Muro K, Chung HC, Shankaran V, Geva R, Catenacci D, Gupta S, et al. Pembrolizumab for patients with PD-L1-positive advanced gastric cancer (KEYNOTE-012): a multicentre, open-label, phase 1b trial. Lancet Oncol. 2016;17(6):717-26.

9. Tran PN, Sarkissian S, Chao J, Klempner SJ. PD-1 and PD-L1 as emerging therapeutic targets in gastric cancer: current evidence. Gastrointest Cancer. 2017;7:1-11.

10. Hegde PS, Karanikas V, Evers S. The where, the when, and the how of immune monitoring for Cancer immunotherapies in the era of checkpoint inhibition. Clin Cancer Res. 2016;22(8):1865-74.

11. Amlot PL, Tahami F, Chinn D, Rawlings E. Activation antigen expression on human T cells. I. Analysis by two-colour flow cytometry of umbilical cord blood, adult blood and lymphoid tissue. Clin Exp Immunol. 1996;105(1):176-82.

12. Lanitis E, Dangaj D, Irving M, Coukos G. Mechanisms regulating T-cell infiltration and activity in solid tumors. Ann Oncol. 2017;28(suppl_12):xii18-32.

13. Das R, Verma R, Sznol M, Boddupalli CS, Gettinger SN, Kluger H, et al. Combination therapy with anti-CTLA-4 and anti-PD-1 leads to distinct immunologic changes in vivo. J Immunol. 2015;194(3):950-9. 
14. Anderson AC, Joller N, Kuchroo VK. Lag-3, Tim-3, and TIGIT: coinhibitory receptors with specialized functions in immune regulation. Immunity. 2016;44(5):989-1004.

15. Hamamoto Y. Complications in advanced or recurrent gastric cancer patients with peritoneal metastasis during and after palliative systemic chemotherapy. Mol Clin Oncol. 2015;3(3):539-42.

16. Ayantunde AA, Parsons SL. Pattern and prognostic factors in patients with malignant ascites: a retrospective study. Ann Oncol. 2007;18(5):945-9.

17. Lim B, Kim C, Kim JH, Kwon WS, Lee WS, Kim JM, et al. Genetic alterations and their clinical implications in gastric cancer peritoneal carcinomatosis revealed by whole-exome sequencing of malignant ascites. Oncotarget. 2016;7(7):8055-66.

18. Sasada T, Kimura M, Yoshida Y, Kanai M, Takabayashi A. CD4+CD25+ regulatory $T$ cells in patients with gastrointestinal malignancies: possible involvement of regulatory T cells in disease progression. Cancer. 2003; 98(5):1089-99.

19. Zhan N, Dong WG, Wang J. The clinical significance of vascular endothelial growth factor in malignant ascites. Tumour Biol. 2016;37(3):3719-25.

20. Wada J, Suzuki H, Fuchino R, Yamasaki A, Nagai S, Yanai K, et al. The contribution of vascular endothelial growth factor to the induction of regulatory T-cells in malignant effusions. Anticancer Res. 2009;29(3):881-8.

21. Neuzillet C, Tijeras-Raballand A, Cohen R, Cros J, Faivre S, Raymond E, et al. Targeting the TGFbeta pathway for cancer therapy. Pharmacol Ther. 2015;147:22-31.

22. Huang $\mathrm{S}$, Xie K, Bucana CD, Ullrich SE, Bar-Eli M. Interleukin 10 suppresses tumor growth and metastasis of human melanoma cells: potential inhibition of angiogenesis. Clin Cancer Res. 1996;2(12):1969-79.

23. Bamias A, Tsiatas ML, Kafantari E, Liakou C, Rodolakis A, Voulgaris Z, et al. Significant differences of lymphocytes isolated from ascites of patients with ovarian cancer compared to blood and tumor lymphocytes. Association of CD3+CD56+ cells with platinum resistance. Gynecol Oncol. 2007;106(1):75-81.

24. Kim HS, Shin SJ, Beom SH, Jung M, Choi YY, Son T, et al. Comprehensive expression profiles of gastric cancer molecular subtypes by immunohistochemistry: implications for individualized therapy. Oncotarget. 2016;7(28):44608-20

25. Mukaka MM. Statistics corner: a guide to appropriate use of correlation coefficient in medical research. Malawi Med J. 2012;24(3):69-71.

26. Tunes-da-Silva G, Klein JP. Cutpoint selection for discretizing a continuous covariate for generalized estimating equations. Comput Stat Data Anal. 2011;55(1):226-35.

27. Fuchs CS, Tomasek J, Yong CJ, Dumitru F, Passalacqua R, Goswami C, et al. Ramucirumab monotherapy for previously treated advanced gastric or gastro-oesophageal junction adenocarcinoma (REGARD): an international, randomised, multicentre, placebo-controlled, phase 3 trial. Lancet. 2014;383(9911):31-9.

28. Parker KH, Beury DW, Ostrand-Rosenberg S. Myeloid-derived suppressor cells: critical cells driving immune suppression in the tumor microenvironment. Adv Cancer Res. 2015;128:95-139.

29. Matte I, Lane D, Laplante C, Rancourt C, Piche A. Profiling of cytokines in human epithelial ovarian cancer ascites. Am J Cancer Res. 2012;2(5):566-80

30. Lukesova S, Vroblova V, Tosner J, Kopecky J, Sedlakova I, Cermakova E, et al. Comparative study of various subpopulations of cytotoxic cells in blood and ascites from patients with ovarian carcinoma. Contemp Oncol (Pozn). 2015;19(4):290-9.

31. Rea IM, McNerlan SE, Alexander HD. CD69, CD25, and HLA-DR activation antigen expression on CD3+ lymphocytes and relationship to serum TNFalpha, IFN-gamma, and sIL-2R levels in aging. Exp Gerontol. 1999;34(1):79-93.

32. $\mathrm{Hu} G$, Wang $\mathrm{S}$. Tumor-infiltrating $\mathrm{CD} 45 \mathrm{RO}(+)$ memory $\mathrm{T}$ lymphocytes predict favorable clinical outcome in solid tumors. Sci Rep. 2017;7(1):10376.

33. Shang B, Liu Y, Jiang SJ. Prognostic value of tumor-infiltrating FoxP3+ regulatory $T$ cells in cancers: a systematic review and meta-analysis. Sci Rep. 2015;5:15179.

34. Santegoets SJ, Welters MJ, van der Burg SH. Monitoring of the Immune Dysfunction in Cancer Patients. Vaccines (Basel). 2016;4(3):29.

35. Wargo JA, Reddy SM, Reuben A, Sharma P. Monitoring immune responses in the tumor microenvironment. Curr Opin Immunol. 2016;41:23-31.

\section{Publisher's Note}

Springer Nature remains neutral with regard to jurisdictional claims in published maps and institutional affiliations.

Ready to submit your research? Choose BMC and benefit from:

- fast, convenient online submission

- thorough peer review by experienced researchers in your field

- rapid publication on acceptance

- support for research data, including large and complex data types

- gold Open Access which fosters wider collaboration and increased citations

- maximum visibility for your research: over $100 \mathrm{M}$ website views per year

At BMC, research is always in progress.

Learn more biomedcentral.com/submissions 